\title{
Evaluation of serum vitamin D levels in elderly patients with subclinical hypothyroidism
}

\author{
Fatma Ucar $^{\mathrm{a} *}$, Sümeyya Akyol ${ }^{\mathrm{b}, \mathrm{c}}$, Gulfer Ozturk ${ }^{\mathrm{a}}$, Zeynep Ginis ${ }^{\mathrm{a}}$, Gonul Erden ${ }^{\mathrm{a}}$, Mine Yavuz Taslipinar ${ }^{\mathrm{a}}$, Abdullah Ercan \\ Arzuhal $^{\mathrm{a}}$, Tuncay Delibasi ${ }^{\mathrm{d}}$
}

\author{
${ }^{a}$ Department of Clinical Biochemistry, Diskapi Yildirim Beyazit Training and Research Hospital, Ankara, Turkey \\ ${ }^{b}$ Turgut Ozal University, Vocational School of Health Sciences, Ankara, Turkey \\ ${ }^{c}$ Department of Biochemistry, Gaziosmanpasa University Medical School, Tokat, Turkey \\ ${ }^{d}$ Department of Endocrinology and Metabolism, Diskapi Yildirim Beyazit Training and Research Hospital, Ankara, Turkey
}

\section{ARTICLE INFO ABSTRACT}

\section{Article History}

Received $\quad 12 / 05 / 2014$

Accepted $\quad 07 / 06 / 2014$

\section{* Correspondence to:}

Fatma Ucar

Department of Clinical Biochemistry,

Diskapi Yildirim Beyazit Training and

Research Hospital, Ankara, Turkey

e-mail: drfucar@gmail.com

\section{Keywords:}

Autoantibodies

Elderly

Subclinical hypothyroidism

Vitamin D

\begin{abstract}
Increasing numbers of researches have indicated the relationship between serum vitamin D level and several autoimmune diseases, because it has important roles in immune regulation. However, the role of vitamin D in the etiopathogenesis of these diseases is not well understood. Similarly, the detailed mechanism of vitamin D action on thyroid hormone metabolism and autoimmune thyroid diseases is still poorly understood. It was recently reported that patients with autoimmune thyroid disease showed lower vitamin D levels. However, there are few studies dealing with vitamin D status in elderly patients with subclinical hypothyroidism. We therefore aimed to investigate vitamin D levels in elderly patients with subclinical hypothyroidism. In the present study, a total of 37 patients ( $>70$ years old) with subclinical hypothyroidism and 40 healthy controls ( $>70$ years old) were retrospectively analyzed. Serum levels of free triiodothyronine (fT3), free thyroxine (fT4) and thyroid-stimulating hormone (TSH) were analyzed by chemiluminescence immunoassay (Centaur XP, Siemens Healthcare Diagnostics Inc., Tarrytown, USA). Serum levels of 25-Hydroxy vitamin D (25-OH D) were measured by chemiluminescence immunoassay (DiaSorin, Liaison, Italy). Serum anti thyroid peroxidase (anti-TPO) and anti thyroglobulin (anti-TG) were measured using a solid-phase, enzyme-labeled, chemiluminenescent sequential immunometric assay (Immulite $2000 \mathrm{XPi}$, Siemens Healthcare Diagnostics Inc., Tarrytown, USA). There was no significant difference between groups in terms of age and gender distribution. Serum TSH, anti-TPO and antiTG levels in elderly patients with subclinical hypothyroidism showed higher values than healthy controls as expected. Serum 25-OH D levels in elderly patients with subclinical hypothyroidism were lower than healthy controls $(p<0.001)$. These data may suggest association between serum 25-OH D levels and subclinical hypothyroidism in elderly patients. Further studies are needed to determine whether vitamin D insufficiency is a casual factor in the etiopathogenesis of subclinical hypothyroidism in elderly patients or rather a consequence of the disease.
\end{abstract}

J. Exp. Clin. Med., 2014; 31:77-80

\section{Introduction}

Vitamin D is the principal regulator of calcium homeostasis and also known as an immune modulator hormone. Vitamin D deficiency is a common public health problem; over a billion people worldwide are vitamin $\mathrm{D}$ deficient or insufficient (Holick and Chen, 2008). It has been recently shown that vitamin D deficiency is associated with various diseases such as cardiovascular disease, cancer, osteoporosis and autoimmune diseases (Vilarrasa et al., 2010). On the other hand, experimental data showed that administration of vitamin D prevented or suppressed some kinds of autoimmune diseases such as encephalomyelitis, arthritis, and inflammatory bowel disease (Deluca and Cantorna, 2001; Ardizzone et al., 2011; Van Belle et al., 2011). Increasing numbers of researches have indicated the relation between serum vitamin D level and several autoimmune diseases because of its important roles in immune regulation (Goswami et al., 2009; Proal et al., 2009; Kivity et al., 2011). However 
the role of vitamin D in the etiopathogenesis of these diseases is not well understood. Furthermore, the detailed mechanism of vitamin $\mathrm{D}$ action on thyroid hormone metabolism and autoimmune thyroid diseases is still poorly understood.

Subclinical hypothyroidism, characterized with elevated serum thyroid-stimulating hormone (TSH) level in the presence of normal levels of free thyroid hormone, is quite common in adult population, especially in women above 60 years of age. Majority of these patients have serum TSH levels ranging between 5 and $10 \mathrm{mIU} / \mathrm{L}$ and also have circulating thyroid autoantibodies (Canaris et al., 2000).

Aging is associated with an increased prevalence of thyroid diseases. Subclinical forms of thyroid diseases, hypothyroidism in particular, are even more frequent (Faggiano et al., 2011; Cooper and Biondi, 2012). Furthermore, elderly people as well as children and young adults are potentially at high risk for vitamin D deficiency (Holick, 2006; Lips et al., 2006; Gordon et al., 2008). It was recently reported that patients with autoimmune thyroid disease had lower vitamin D levels (Tamer et al., 2011). However, there are very few studies examining vitamin D status especially in elderly patients with subclinical hypothyroidism. In the present study, we therefore aimed to investigate vitamin D levels in elderly patients with subclinical hypothyroidism.

\section{Material and methods}

\section{Subjects}

A total of 77 elder subjects ( $\geq 70$ years old) were included in this study. Group I: Control group included 40 healthy individuals. Control subjects who had normal thyroid function, with no history of chronic diseases and use of vitamin D supplements. Group II included 37 patients who were diagnosed as subclinical hypothyroidism. Subclinical hypothyroid patients had normal serum free thyroxine (fT4) and free triiodothyronine (fT3) levels but with serum TSH levels elevated to the range of 5-20 mIU/L. All subjects were matched on age and gender. This retrospective study was approved by our local Ethical Committee.

\section{Blood measurements}

25-Hydroxy vitamin D (25-OH D) is the preferred diagnostic test for the demonstration of vitamin D deficiency. Vitamin $\mathrm{D}$ deficiency was determined as serum levels of 25-OH D less than $20 \mathrm{ng} / \mathrm{ml}$ (Ralston et al., 2011). Serum levels of 25$\mathrm{OH} \mathrm{D}$ were measured by chemiluminescence immunoassay (DiaSorin, Liaison, Italy). Serum fT3, fT4 and TSH were analyzed by chemiluminescence immunoassay (Centaur XP, Siemens Healthcare Diagnostics Inc., Tarrytown, USA). Serum thyroid peroxidase antibody (anti-TPO) and thyroglobulin antibody (anti-TG) were measured by using a solid-phase, enzyme-labeled, chemiluminenescent sequential immunometric assay according to standard protocols (Immulite 2000 XPi, Siemens Healthcare Diagnostics Inc., Tarrytown, USA).

\section{Data analysis}

All statistical analyses were carried out using the SPSS statistical package software version 15.0 (SPSS Inc., Chicago, Illinois, USA). The Kolmogorov-Smirnov test was used to determine the normality of distribution for numerical variables. Comparisons between the two study groups were made using either independent T-test or the Mann-Whitney U-test, where appropriate. Numerical variables were expressed as mean \pm standard deviation (SD). A p-value of less than 0.05 was considered to indicate statistical significance.

\section{Results}

The mean values \pm SD of all laboratory parameters, age and sex distribution in all groups are shown in Table 1. All serum TSH levels in subclinical hypothyroid patients were above $5 \mathrm{mIU} / \mathrm{L}$. On the other hand, all of serum TSH, fT3 and fT4 levels in control group were within normal range. Mean fT3 and fT4 values were similar in both patients and controls. There was no significant difference between groups in terms of age and gender distribution.

Serum TSH, anti-TPO and anti-TG levels in elderly patients with subclinical hypothyroidism revealed higher values than healthy controls $(\mathrm{p}<0.001, \mathrm{p}<0.05$ and $\mathrm{p}<0.05$, respectively), as expected. Serum 25-OH D levels in elderly patients with subclinical hypothyroidism were lower than healthy controls $(p<0.001)$. Box plot for serum 25-OH D levels of each group is shown in Figure 1. When compared serum 25-OH D levels according to genders, they were insignificantly lower than those of male in subclinical hypothyroid patients (Table 2).

\begin{tabular}{lccc} 
Table 1. Demographic characteristics and laboratory parameters \\
of study and control groups \\
& $\begin{array}{c}\text { Study group } \\
(\mathbf{n}=\mathbf{3 7})\end{array}$ & $\begin{array}{c}\text { Control group } \\
(\mathbf{n}=\mathbf{4 0})\end{array}$ & p-value \\
\hline Gender (Female/Male) & $30 / 7$ & $32 / 8$ & $>0.05$ \\
Age (years) & $75.3 \pm 4.4$ & $74.6 \pm 4.4$ & $>0.05$ \\
TSH (mIU/L) & $17.19 \pm 19.31$ & $1.58 \pm 1.22$ & $<0.001$ \\
fT3 (ng/dL) & $2.75 \pm 0.35$ & $2.69 \pm 0.27$ & $>0.05$ \\
fT4 (pg/mL) & $1.02 \pm 0.22$ & $1.04 \pm 0.17$ & $<0.05$ \\
Anti-TPO (IU/mL) & $84.45 \pm 164.34$ & $2.08 \pm 4.60$ & $<0.05$ \\
Anti-TG (IU/mL) & $53.58 \pm 100.32$ & $5.77 \pm 11.77$ & $<0.05$ \\
25-OH D (ng/mL) & $13.12 \pm 9.60$ & $43.42 \pm 11.96$ & $<0.001$ \\
\hline Data presented as mean \pm SD & &
\end{tabular}

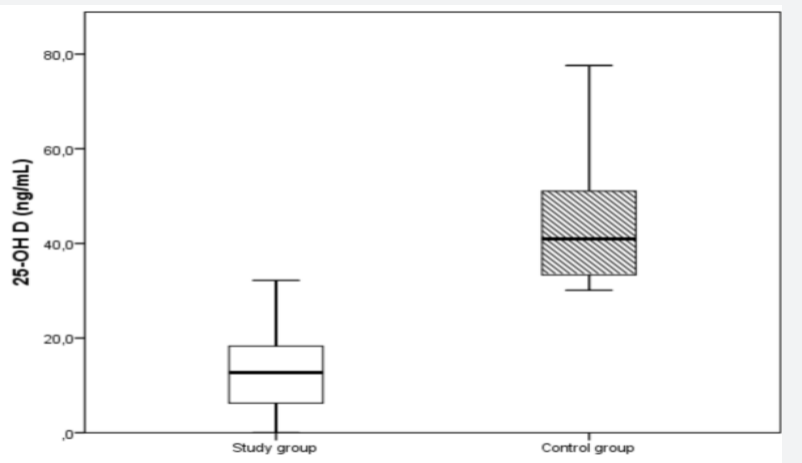

Fig. 1. Box plots graphs of 25-OH D values of study group and control group

\section{Discussion}

In this study, we observed significant lower vitamin D levels in elderly subclinic hypothyroid patients. Vitamin D insufficiency may be associated with subclinical hypothyroidism in elderly people. However, the explanation of these findings is not clear, low levels of vitamin D may 
Table 2. Mean \pm SD of serum 25-OH D, Anti-TPO, Anti-TG levels in subclinical hypothyroid patients according to gender

\begin{tabular}{lccc} 
& $\begin{array}{c}\text { Female } \\
(\mathbf{n}=\mathbf{3 0})\end{array}$ & $\begin{array}{c}\text { Male } \\
(\mathbf{n}=7)\end{array}$ & p-value \\
\hline 25-OH D $(\mathbf{n g} / \mathbf{m L})$ & $11.79 \pm 9.68$ & $18.83 \pm 7.27$ & $>0.05$ \\
Anti-TPO $(\mathbf{I U} / \mathbf{m L})$ & $102.1 \pm 178.08$ & $8.85 \pm 23,43$ & $<0.05$ \\
Anti-TG $(\mathbf{I U} / \mathbf{m L})$ & $64.58 \pm 108.5$ & $6.42 \pm 17$ & $<0.05$ \\
\hline
\end{tabular}

be a casual factor in the etiopathogenesis of subclinical hypothyroidism in elderly patients or rather a consequence of the disease.

Several studies on vitamin $\mathrm{D}$ have shown that vitamin $\mathrm{D}$ deficiency and insufficiency is still one of the major public health problems, especially in developing countries (Hochberg et al., 2002; Holick, 2007; Van Schoor and Lips, 2011). Vitamin D has potent immunomodulatory effects and plays important roles in the pathogenesis of autoimmune diseases. Furthermore, vitamin D supplementation prevented the onset and/or development of several kinds of autoimmune diseases in humans and animal models (Baeke et al., 2010). The results of the studies show that 1.25-dihydroxyvitamin D3 can either prevent or markedly suppress experimental autoimmune encephalomyelitis, rheumatoid arthritis, systemic lupus erythematosus, type I diabetes, and inflammatory bowel disease (Deluca and Cantorna, 2001).

Autoimmune thyroid diseases are among the most common autoimmune diseases (Shapira et al., 2010; Shoenfeld et al., 2008a). The pathogenesis of autoimmune thyroid diseases, like other autoimmune diseases, is multifactorial, comprising genetic, immune, environmental and hormonal influences such as vitamin D (Shoenfeld et al., 2008b; Gershwin, 2008). Few studies have been conducted in order to find any significant association between the levels of vitamin D and hypothyroidism and to determine whether vitamin D deficiency involves in the pathogenesis of hypothyroidism or rather a consequence of the disease and those that yielded conflicting results.

In the current study our data indicate an association between vitamin $\mathrm{D}$ deficiency and thyroid dysfunction in elderly patients, specifically when caused by an autoimmune disease. Serum TSH, anti-TPO and anti-TG levels in elderly patients with subclinical hypothyroidism showed expected higher values than healthy controls, however serum $25-\mathrm{OH}$ $\mathrm{D}$ levels in elderly patients with subclinical hypothyroidism were lower than healthy controls. When compared serum 25-
$\mathrm{OH} \mathrm{D}$ levels according to genders, they were insignificantly lower than those of male in subclinical hypothyroid patients. In contrast to our study, Aljohani et al. (2013) have demonstrated that patients with subclinical hypothyroidism had higher serum 25-OH D levels than controls and higher vitamin D levels were associated with lower fT3 in the patients. Another study was previously reported that high vitamin D status in younger individuals is associated with low circulating TSH (Chailurkit, 2013). However, our study population was elderly patients with subclinical hypothyroidism, not younger individuals.

Tamer et al. (2013) showed that patients with Hashimoto's thyroiditis had lower 25-OH D levels than healthy subjects in a younger population with a mean age of 34.8 years and also vitamin D insufficiency was more common in patients with Hashimoto's thyroiditis than in healthy people. Similar to our study, Bozkurt et al. (2013) reported that serum 25$\mathrm{OH}$ D levels of patients with Hashimoto's thyroiditis were significantly lower than controls and severity of vitamin D deficiency correlated with duration of Hashimoto's thyroiditis, thyroid volume and antibody levels. Distinct from these studies we excluded the subclinical hypothyroidism patients whose ages were not higher than 70 years. In another study, Shin et al (2014) demonstrated a negative association between 25-OH D and anti-TPO levels, and also indicated that the low 25-OH D level was a possible risk factor of antiTPO positivity.

The authors acknowledge several limitations. Seasonal variations and body mass index are other two important confounding factors. However, we have not evaluated these factors in our study. Furthermore, clinical parameters associated with thyroid disease, such as cigarette smoking, were not available in this study.

In conclusion, obtained data may indicate the association between serum 25-OH D levels and subclinical hypothyroidism in elderly patients. Low levels of vitamin D were associated with the presence of antithyroid antibodies and abnormal thyroid functions. Further studies are needed to determine whether vitamin D insufficiency is a casual factor in the etiopathogenesis of subclinical hypothyroidism in elderly patients or rather a consequence of the disease.

\section{Declaration of conflicting interests}

The authors declared no conflicts of interest with respect to the authorship and/or publication of this article.

\section{REFERENCES}

Aljohani, N.J., Al-Daghri, M.N., Al-Attas, O.S., Alokail, M.S., Alkhrafy, K.M., Al-Othman, A., Yakout, S., Alkabba, A.F., Al-Ghamdi, A.S., Almalki, M., Buhary, B.M., Sabico, S., 2013. Differences and associations of metabolic and vitamin D status among patients with and without sub-clinical hypothyroid dysfunction. BMC Endocr Disord. 13, 31. doi: 10.1186/1472-6823-13-31.

Ardizzone, S., Cassinotti, A., Bevilacqua, M., Clerici, M., Porro G.B., 2011. Vitamin D and inflammatory bowel disease. Vitam Horm. 86, 367377. doi: 10.1016/B978-0-12-386960-9.00016-2.

Baeke, F., Takiishi, T., Korf, H., Gysemans, C., Mathieu, C., 2010. Vitamin D: Modulator of the immune system. Curr. Opin. Pharmacol. 10, 482-496. doi: 10.1016/j.coph.2010.04.001.

Bozkurt, N.C., Karbek, B., Ucan, B., Sahin, M., Cakal, E., Ozbek, M., Delibas1, T., 2013. The association between severity of vitamin D deficiency and Hashimoto's thyroiditis. Endocr. Pract. 19, 479-484. doi: 10.4158/EP12376.OR.

Canaris, G.J., Manowitz, N.R., Mayor, G., Ridgway, C., 2000. The Colorado thyroid disease prevalence study. Arch. Intern. Med. 160, 526-534.

Chailurkit, L.O., Aekplakorn, W., Ongphiphadhanakul, B., 2013. High vitamin D status in younger individuals is associated with low circulating thyrotropin. Thyroid. 23, 25-30. doi: 10.1089/thy.2012.0001.

Cooper, D.S., Biondi B., 2012. Subclinical thyroid disease. Lancet. 379, 1142-1154. doi: 10.1016/S0140-6736(11)60276-6.

Deluca, H.F., Cantorna, M.T., 2001. Vitamin D: its role and uses in immunology. Faseb. J. 15, 2579-2585.

Faggiano, A., Del Prete, M., Marciello, F., Marotta, V., Ramundo, V., Colao, A. 2011. Thyroid diseases in elderly. Minerva Endocrinol. 36, 211- 
231.

Gershwin, M.E., 2008. The mosaic of autoimmunity. Autoimmun Rev. 7, 161-163. doi: 10.1016/j.autrev.2007.11.021.

Gordon, C.M., Feldman, H.A., Sinclair, L., Williams, A.L., Kleinman, P.K., Perez-Rossello, J., Cox, J.E. 2008. Prevalence of vitamin D deficiency among healthy infants and toddlers. Arch. Pediatr. Adolesc. Med. 162, 505-512. doi: 10.1001/archpedi.162.6.505.

Goswami, R,, Marwaha, R.K., Gupta, N., Tandon, N., Sreenivas, V., Tomar, N., Ray, D., Kanwar, R., Agarwal, R., 2009. Prevalence of vitamin D deficiency and its relationship with thyroid autoimmunity in Asian Indians: A community-based survey. Br. J. Nutr. 102, 382-386. doi: 10.1017/S0007114509220824.

Hochberg, Z., Bereket, A., Davenport, M., Delemarre-Van de Waal, H.A., De Schepper, J., Levine, M.A., Shaw, N., Schoenau, E., van Coeverden, S.C., Weisman, Y., Zadik, Z., 2002. Consensus development for the supplementation of vitamin D in childhood and adolescence. Horm. Res. 58, 39-51.

Holick, M.F., 2006. High prevalence of vitamin D inadequacy and implications for health. Mayo. Clin. Proc. 81, 353-373.

Holick, M. F., 2007. Vitamin D deficiency. N. Engl. J. Med. 357, 266-281.

Holick, M.F., Chen, T.C. 2008. Vitamin D deficiency a worldwide problem withhealth consequences. Am. J. Clin. Nutr. 87, $10805-10868$.

Kivity, S., Agmon-Levin, N., Zisappl, M., Shapira, Y., Nagy, E.V., Dankó, K., Szekanecz, Z., Langevitz, P., Shoenfeld, Y., 2011. Vitamin D and autoimmune thyroid diseases. Cell Mol. Immunol. 8, 243-247. doi: 10.1038/cmi.2010.73.

Lips, P., Hosking, D., Lippuner, K., Norquist, J.M., Wehren, L., Maalouf, G., Ragi-Eis, S., Chandler, J, 2006. The prevalence of vitamin D inadequacy amongst women with osteoporosis: an international epidemiological investigation. J. Intern. Med. 260, 245-254.

Proal, A.D., Albert, P.J., Marshall, T.G., 2009. Dysregulation of the vitamin D nuclear receptor may contribute to the higher prevalence of some autoimmune diseases in women. Ann. N. Y. Acad. Sci. 1173, 252-259. doi: 10.1111/j.1749-6632.2009.04672.x.

Ralston, S.H.1., Binkley, N., Boonen, S., Kiel „D.P., Reginster, J.Y., Roux, C., Chen, L., Rosenberg, E., Santora, A., 2011. FOCUS-D (FOSAVANCE vs. Standard Care-Use and Study of Vitamin D) Trial. Randomized trial of alendronate plus vitamin D3 versus standard care in osteoporotic postmenopausal women with vitamin D insufficiency. Calcif. Tissue. Int. 88, 485-494. doi: 10.1007/s00223-011-9482-4.

Shapira, Y., Agmon-Levin, N., Shoenfeld, Y., 2010. Geoepidemiology of autoimmune diseases. Autoimmunity. 8, 468-476. doi: 10.1038/nrrheum.2010.86.

Shin, D.Y., Kim, K.J., Kim, D., Hwang, S., Lee, E.J., 2014. Low serum vitamin D is associated with anti-thyroid peroxidase antibody in autoimmune ahyroiditis. Yonsei. Med. J. 55, 476-481. doi: 10.3349/ymj.2014.55.2.476.

Shoenfeld, Y., Selmi, C., Zimlichman, E., Gershwin, M.E., 2008a. The autoimmunologist: Geoepidemiology, a new center of gravity, and prime time for autoimmunity. J Autoimmun. 31, 325-330. doi: 10.1016/j.jaut.2008.08.004.

Shoenfeld, Y., Blank, M., Abu-Shakra, M., Amita,l H., Barzilai, O., Berkun, Y., Bizzaro, N., Gilburd, B., Zandman-Goddard, G., Katz, U., Krause, I., Langevitz, P., Mackay, I.R., Orbach, H., Ram, M., Sherer, Y., Toubi, E., Gershwin, M.E. 2008b. The mosaic of autoimmunity: Prediction, autoantibodies, and therapy in autoimmune diseases-2008. Isr. Med. Assoc. J. 10, 13-19.

Tamer,G., Arik, S., Tamer, I., Coksert, D., 2011. Relative vitamin D insufficiency in Hashimoto's thyroiditis. Thyroid. 21, 891-896. doi: 10.1089/ thy.2009.0200.

Van Belle, T.L., Gysemans, C., Mathieu, C., 2011. Vitamin D in autoimmune, infectious and allergic diseases: A vital player? Best. Pract. Res. Clin. Endocrinol. Metab. 25, 617-632. doi: 10.1016/j.beem.2011.04.009.

Van Schoor, N.M., Lips, P., 2011. Worldwide vitamin D status. Best. Pract. Res. Clin. Endocrinol. Metab. 25, 671-680. doi: 10.1016/j. beem.2011.06.007.

Vilarrasa, N., Vendrell, J., Maravall, J., Elío, I., Solano, E., San José, P., García, I., Virgili, N., Soler, J., Gómez , J.M., 2010. Is plasma 25(OH) $\mathrm{D}$ related to adipokines, inflammatory cytokines and insulin resistance in both a healthy and morbidly obese population? Endocrine. 38 , 235-42. doi: 10.1007/s12020-010-9379-4. 